\title{
Test my product using sine or random?
}

\section{John Van Baren}

Vibration Research, Jenison, MI, United States

E-mail:vrsales@vibrationresearch.com

Received 3 November 2017; accepted 13 November 2017

DOI https://doi.org/10.21595/vp.2017.19354

\begin{abstract}
As you know, in the vibration world, there are quite a few test "types" to which you can expose your product. The major choices are Sine, Random, Classical Shock, Transient Shock, Field Recorded Time History, Sine-on-Random, Random-on-Random and Sine-and- Random-onRandom. Frequently, our customers will request advice on which of these types of test to run on their product, and in particular, how to choose between the two most common test types: sine or random. Their desire is to know which test, sine or random, is best to most quickly pinpoint flaws in their product. If they can only run one test, either sine or random, which should it be? Recently, I received an even more specific request from a customer. This customer (Don) presented both a sine test and a random test and wanted to know, given both a sine test and a random test, how he could determine which is the most severe? Let's take a look at the two tests and decide how to answer to his question. Here is Don's question: How would the following specifications compare with regard to amplitude/severity?
\end{abstract}

Keywords: sine, random, amplitude.

\section{Introduction}

\subsection{Sine test}

$3.5 \mathrm{G}$ at 5 to $50 \mathrm{~Hz}$.

$1.5 \mathrm{G}$ at 50 to $300 \mathrm{~Hz}$.

Limit vibration to 0.4 inches double amplitude.

Test all axes at the same level.

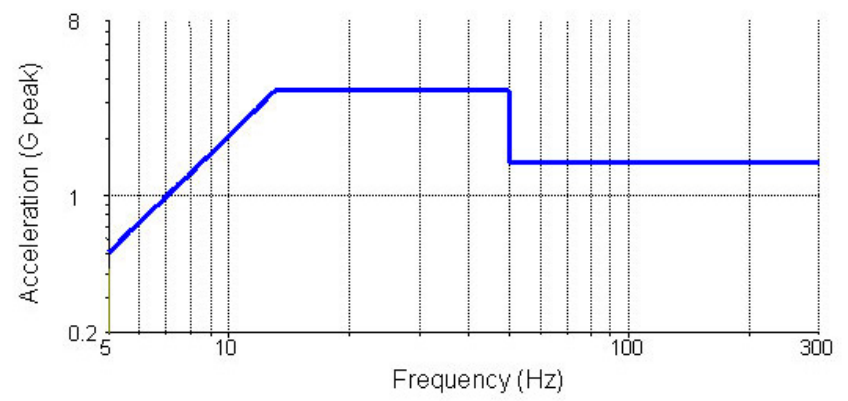

Fig. 1. Sample Sine test profile

\subsection{Random test}

$0.01500 \mathrm{G}_{2} / \mathrm{Hz}$ from $10 \mathrm{~Hz}$ to $0.01500 \mathrm{G}^{2} / \mathrm{Hz}$ at $40 \mathrm{~Hz}$.

$0.01500 \mathrm{G}_{2} / \mathrm{Hz}$ at $40 \mathrm{~Hz}$ to $0.00015 \mathrm{G}^{2} / \mathrm{Hz}$ at $500 \mathrm{~Hz}$.

Results in $1.05 \mathrm{G}$ rms.

Test all axes at the same level.

\subsection{John's response}

This is a very valid and interesting question. Why? Because the answer is not obvious. The 
sine vibration is measured in $\mathrm{G}$ peak, while the random vibration is measured as $\mathrm{G}$ rms, with the peak $\mathrm{G}$ levels typically left to a statistical assumption. A quick calculation tells us that the random test, which can have peak values up to 4 or even 5 times the RMS level, will apply $4 \times 1.05 \mathrm{G} \mathrm{rms,}$ or $4.20 \mathrm{G}$ peak to our product. Since the sine test is only $3.5 \mathrm{G}$ peak, we would expect the random test to be more damaging, right?

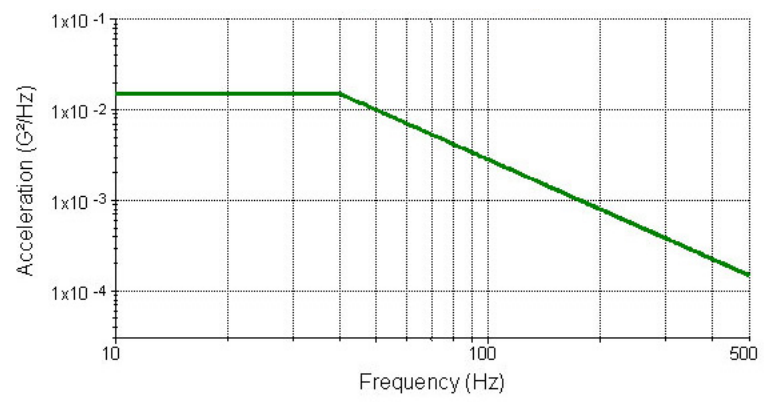

Fig. 2. Sample Random test profile

\section{Analysis}

Looking for support from some equations, let's make some reasonable assumptions: 1) failures due to vibration are caused at the peak $G$ level seen by the product, 2) most products have resonances at one or more frequencies, and 3 ) at these resonances the vibration levels applied to the product are amplified by the $Q$ factor of the resonance.

Following this train of logic, we conclude that the failures will occur when the vibration is at one of the resonant frequencies. Now when we use a sine vibration, the full vibration levels are concentrated at the resonant frequency, and the vibration levels are simply amplified by the amplification factor $Q$ :

$A_{\text {product, sine }}=Q \times A_{\text {control,sine }}$.

When we use a random vibration, it is not so simple because not all of the vibration is amplified by the resonance. Let's further assume the resonance has a $Q$ factor of 5 or more. In that case the resonance will act as an amplifying band-pass filter with amplification equal to $Q$, and a bandwidth equal to $\Delta f$, where:

$Q=\frac{f_{n}}{\Delta f}$

where $f_{n}$ is resonant frequency, $\Delta f$ is half-power bandwidth of the resonance.

It will also be helpful to refer to the following relationship, which tells us that, for a given RMS level, the PSD level is inversely proportional to the full bandwidth of the random spectrum. In more general terms, a more concentrated random vibration will have a higher PSD value:

$P S D_{\text {control }}=\frac{A_{\text {control, } r m s^{2}}}{\Delta F}$

where $\Delta F$ is full bandwidth of the random PSD, Eq. (3) applies to flat spectrum only.

A random test is defined in terms of a PSD, which is an amplitude-squared measure, so at the resonant frequency the PSD levels of the product will be amplified by $Q^{2}$. Since the resonance acts as an amplifying band-pass filter, we can approximate the vibration levels at the product by looking at just the energy at the resonant frequency that passes through and is amplified by the 
resonance (again assuming the random waveform has up to 4 sigma peaks):

$$
\begin{aligned}
& A_{\text {product, peak }}=4 \times A_{\text {product,rms }}=4 \times\left[P S D_{\text {product }} \times \Delta f\right]^{\frac{1}{2}} \\
& \quad=4 \times\left[Q_{2} \times P S D_{\text {control }} \times \Delta f\right]^{\frac{1}{2}}=4 \times\left[P S D_{\text {control }} \times Q f_{n}\right]^{\frac{1}{2}} .
\end{aligned}
$$

From Eq. (4) we note three features of the peak amplitude for a random test:

1. It is proportional to only the square root of $Q$ ! As a result, a high- $Q$ resonance will result in a more severe test in Sine than it will in Random, if all other parameters are equal.

2. It is proportional to the square root of the resonant frequency, $f_{n}$, so the higher the resonant frequency, the higher the peak values in the output.

3. Referring back to Eq. (3), we also note that the more concentrated the random vibration is, the higher will be the peak vibration levels.

Now we can also compare the peak vibration levels on the product for both Sine and Random tests by comparing Eq. (1) and (3) with Aproduct, sine = Aproduct, peak, random:

$A_{\text {control,sine }}=\frac{A_{\text {product, sine }}}{Q}=\frac{A_{\text {product,peak, random }}}{Q}=4 \times\left[\frac{f_{n} \times P S D_{\text {control }}}{Q}\right]^{\frac{1}{2}}$.

With this we have an equation with which, given a value for the $Q$ factor, we can compare a sine test with a random test. From this result, shown in Fig. 3, we see that the Sine test from Fig. 1 is much more severe than the random test from Fig. 2. Only in the case of a resonance at $50 \mathrm{~Hz}$, where the sine test steps down in amplitude, with $Q=5$ does the random test level come close the sine test level. So, the equations are telling us that the Sine test will be more severe than the Random test!

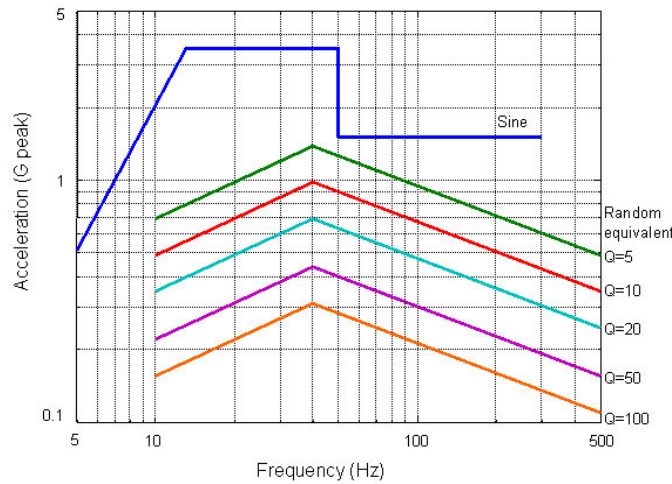

Fig. 3. Comparison of the Sine equivalents of the Random profile, with various $Q$. Note that for higher $Q$, the Sine equivalent of the Random profile has a lower amplitude

\section{Application}

At the time Don asked his question, John was at Sperry Marine in Charlottesville, VA, setting up for some equipment installation training with Dave Maxwell and Joe Reisinger. This was a great time to demonstrate and test for the differences! As a single test is worth a thousand opinions, we set up to run both the random test and the sine test on a slip plate. The slip plate had two elements mounted on it, each with different resonant frequencies. The elements were aluminum masses attached by threaded rods of different lengths and thicknesses, with accelerometers mounted on the mass at the top of each rod connected to channels 4 and 6 .

What we needed to do for the comparison was run the tests, and then look at the G levels seen. 
We knew they would be $3.5 \mathrm{G}$ peak for the sine test at the Control point, and, making a 4 sigma peak assumption, we expected to find $4.2 \mathrm{G}$ peak at the Control point for the random test also. The two plots in Figs. 4 and 5 show the controlled test along with the response data for the two vertical rods with masses attached.

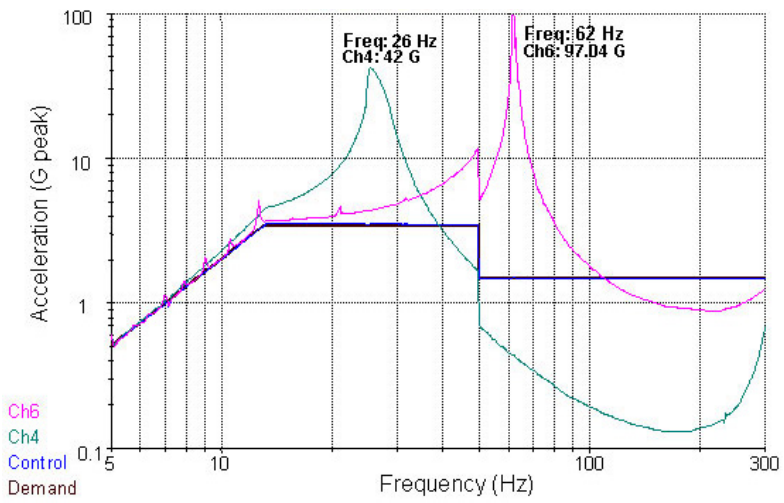

Fig. 4. Sine test results

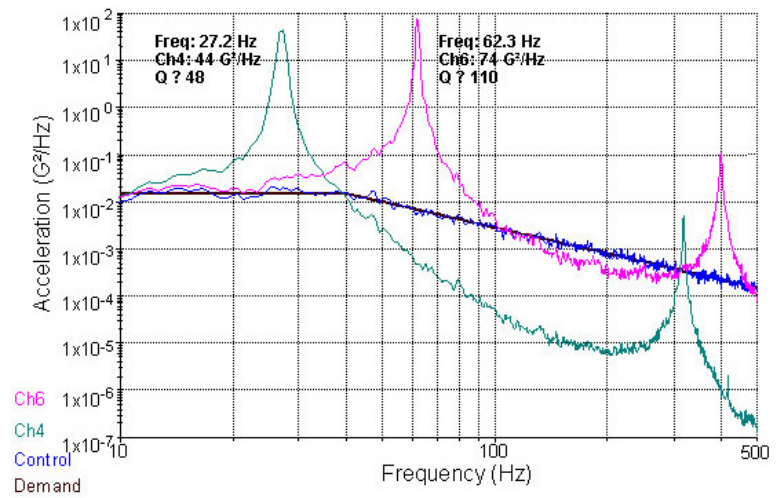

Fig. 5. Random test results

While running the tests, we also simultaneously streamed the accelerometer data to the hard disk drive for later analysis. This allowed us to be able to make a direct comparison of the peak $\mathrm{G}$ levels for each of the tests. As expected, at the Control point, the peak G levels for the Sine test were of course $3.5 \mathrm{G}$ peak and $1.5 \mathrm{G}$ peak. The Random test levels at the Control point were $1.05 \mathrm{G} \mathrm{rms}$, as expected, and $4.8 \mathrm{G}$ peak, which is a little bit higher than the 4 sigma peaks we predicted, but not unusual for a Gaussian random vibration (Fig. 6).

Now, as we were running the test, we observed the resonant elements mounted to the slip table going berserk! This also showed up on the controller plots (Figs. 4 and 5), which showed much larger accelerations on channels 4 and 6 than measured at the Control point. What could be going on here? Recall from Eqs. (1) and (4) above, that when we have resonances in the product, they will amplify the vibration levels. The accelerometers monitoring our two resonant elements show the resonance frequencies are $27 \mathrm{~Hz}$ and $62 \mathrm{~Hz}$, right in the middle of our test range!

Let us examine the time data for those accelerometers, which we had conveniently streamed to the hard disk drive, and see what is happening at the resonant elements. This may shed more light on the test equivalences. First looking at the frequency response for the $\mathrm{Ch} 4$ accelerometer (Fig. 5), we estimate a $Q$ factor of 48 and a resonant frequency of $27 \mathrm{~Hz}$. However, we note that the bandwidth, $\Delta f=f_{n} / Q=0.6 \mathrm{~Hz}$, which is less than the frequency resolution of the PSD plot (using 800 lines). This is a good indication that the $Q$ factor estimated from this PSD will be 
underestimated. Since we had recorded the time waveforms to disk, we are able to load these waveforms into Matlab TM and use high resolution spectral post-processing (13000 lines) to zoom in on the peaks. With this more accurate estimate, we find the $Q$ level to be 140. Computing using Eq. (4), we expect a peak level of $4 \times[0.015 \times 140 \times 27]^{1 / 2}$, or $30 \mathrm{G}$ peak. Fig. 7 shows the actual acceleration levels on $\mathrm{Ch} 4$, where we find the peak $\mathrm{G}$ level was $28 \mathrm{G}$.

Next looking at the frequency response for the Ch6 accelerometer (Fig. 5) we estimate a $Q$ factor of 110 and a resonant frequency of $62 \mathrm{~Hz}$. Again, we find the bandwidth, $0.6 \mathrm{~Hz}$, to be less than the frequency resolution of the PSD plot, so we need to post-process this recorded waveform to obtain a more accurate estimate of the Q factor. Using 13000 lines of resolution we estimate the $\mathrm{Q}$ factor to be 200. Computing using Eq. (4), we expect to see peak levels of $4 \times[0.00673 \times 200 \times 62]^{1 / 2}$, or $37 \mathrm{G}$ peak. Fig. 8 shows the actual acceleration levels on Ch6, where we find the peak $\mathrm{G}$ level was $42 \mathrm{G}$.

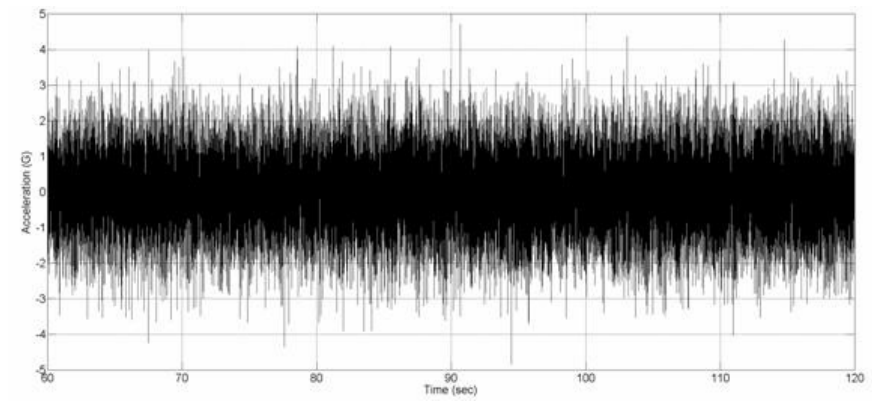

Fig. 6. The random test vibration levels measured at the control point

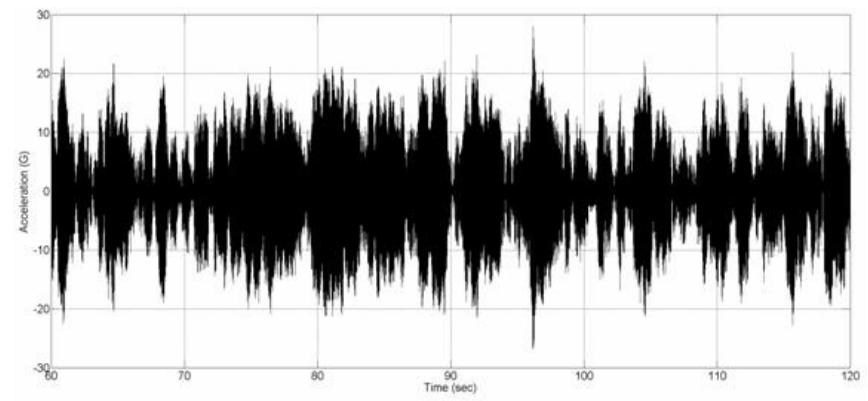

Fig. 7. The random test vibration levels measured on $\mathrm{Ch} 4(27 \mathrm{~Hz}$ resonance)

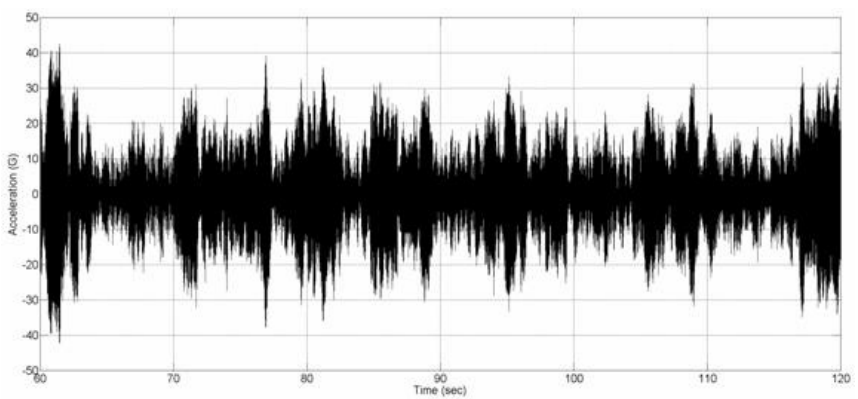

Fig. 8. The random test vibration levels measured on $\mathrm{Ch} 6(63 \mathrm{~Hz}$ resonance $)$

\section{Observations}

Let's make a Table 1 of the results, so we can get the whole picture.

From these results we find that, at the control point, the random test has higher peak G levels 
than the sine test. This would lead us to believe that the random test was the more severe test. However, at the resonant points the sine test has higher peak $\mathrm{G}$ levels than the random test, so from the product's perspective the sine test was the more severe test.

Table 1. Results

\begin{tabular}{|c|c|c|c|c|c|}
\hline & $Q$ & $\begin{array}{c}\text { Sine } \\
(\mathrm{G} \mathrm{peak})\end{array}$ & $\begin{array}{c}\text { Random } \\
(\mathrm{G} \mathrm{rms})\end{array}$ & $\begin{array}{c}\text { Random } \\
(\mathrm{G} \text { peak })\end{array}$ & $\begin{array}{c}\text { Predicted (Eq. (4)) } \\
(\mathrm{G} \mathrm{peak})\end{array}$ \\
\hline Control Point & & $3.5 \mathrm{G}$ & $1.05 \mathrm{G}$ & $4.8 \mathrm{G}$ & $4.2 \mathrm{G}$ \\
\hline Ch4 (27 Hz Resonance) & 140 & $42 \mathrm{G}$ & $8.35 \mathrm{G}$ & $28 \mathrm{G}$ & $30 \mathrm{G}$ \\
\hline Ch6 (62 Hz Resonance) & 200 & $97 \mathrm{G}$ & $10.9 \mathrm{G}$ & $42 \mathrm{G}$ & $37 \mathrm{G}$ \\
\hline
\end{tabular}

You may also notice that the sine resonance peak values were not as high as would be predicted by the $Q$ factor. By reviewing the recorded time data for $\mathrm{Ch} 4$ we are able to determine that, at the $27 \mathrm{~Hz}$ resonance, the sine sweep passed through the resonance too quickly to fully excite the resonance. Repeating the test using a slower sweep rate, or a resonance search-and-dwell feature, would allow us to more accurately find the resonance frequency and $Q$ factor. From this we can conclude that for high $Q$ values, to get the full $Q$ amplification effects of a sine test, you must either sweep slowly or do a resonance dwell at each resonance.

By examining the recorded data for Ch6 we are also able to determine that at the $62 \mathrm{~Hz}$ resonance the acceleration levels were so high that they exceeded the accelerometer's measurement capacity, and therefore the measured waveform was saturated well below the actual acceleration levels. This is an important point, because quite often accelerometers are sized based on the test profile's acceleration level, while the resonances may see $10 \times$ or $100 \times$ the profile acceleration level. Repeating the test using a higher capacity accelerometer is required to accurately measure the acceleration levels at that resonance.

The actual random peak values are a little different from the predicted values, but this is to be expected due to the random nature of the waveform. Each random test you run will be different from any other random test, so the peak values will vary from test to test, and even over different time intervals within a single test.

\section{Conclusions}

The relative severity of a sine test and a random test will vary depending on your product's resonant frequencies and Qs. In general, when sine and random tests have the same peak vibration levels at the control point, the product will see higher vibration levels with a sine test than with a random test due to the resonances in the product.

Eq. (5) gives you way to convert a random PSD into an approximately equivalent sine peak acceleration, as long as you know the resonant frequencies and $Q$ factors of the product.

However, you must also consider that sine tests only excite a single product resonance at a time, so a sine test will not test the interaction between two resonances in your product. Since a random test excites the full frequency range all at the same time, it can be used to find problems resulting from the interaction between two resonances.

\section{References}

[1] SineVIEW Test Module, Vibration Research Corporation. http://www.vibrationresearch.com /sine.html, 2017.

[2] RandomVIEW Test Module, Vibration Research Corporation. http://www.vibrationresearch.com /random.html, 2017. 